\title{
Demand Side Management of Photovoltaic-Battery Hybrid System
}

\author{
Zhou Wu, Henerica Tazvinga, and Xiaohua Xia \\ Department of Electrical Electronic and Computer Engineering, University of Pretoria, \\ Pretoria, South Africa e-mail: wuzhsky@gmail.com, henerica.tazvinga, xxia@up.ac.za
}

\begin{abstract}
In the electricity market, customers have many choices to reduce electricity cost if they can economically schedule their power consumption. Renewable hybrid system, which can explore solar or wind sources at low cost, is a popular choice for this purpose nowadays. In this paper optimal energy management for a grid-connected photovoltaic-battery hybrid system is proposed to sufficiently explore solar energy and to benefit customers at demand side. The management of power flow aims to minimize electricity cost subject to a number of constraints, such as power balance, solar output and battery capacity. With respect to demand side management, an optimal control method (open loop) is developed to schedule the power flow of hybrid system over 24 hours, and model predictive control is used as a closed-loop method to dispatch the power flow in real-time when uncertain disturbances occur. In these two kinds of applications, optimal energy management solutions can be obtained with great cost savings and robust control performance.

Keywords: solar energy, renewable hybrid system, distributed generation, demand side management, optimal control
\end{abstract}

\section{Introduction}

Renewable energy (RE) sources, including wind, solar and their hybrid systems, have become attractive options of providing energy globally for reasons such as low cost, no pollutant emission, energy security, easy accessability and reduction fossil fuel consumption $[1,2,3,4]$. Photovoltaic (PV) array, which is the main technology to convert solar energy into electric power, can be stand-alone installed for providing electricity in some remote areas or be 
connected to the grid for selling power generated. Because of instantaneous and unstable nature of solar energy, PV usually works with battery storage to provide continuous and stable power, i.e., the PV-battery hybrid system. Battery storage can reduce the risk of PV's intermittent power supply, and always ensure demand satisfaction. Generally, grid-connected PV systems without battery storage do not require sophisticated management strategies. Prioritizing use of PV power is the only rule when the PV power is less than the load demand. In contrast, battery storage brings more challenges to energy management, as more complicated scenarios must be considered, such as charging the battery from the grid or PV and discharging when necessary. As a result, controllers are required for hybrid PV-battery systems, such that the performance of solar usage can be significantly enhanced and the grid regulation can be improved in terms of safety and efficiency.

For grid-connected hybrid PV-battery systems, the changing electricity price, the timing of power transaction, and the mismatch between solar power generation and load demand are main challenges in application [5, 6]. From the perspective of demand side management (DSM), solar energy or grid power may be stored when the PV can generate surplus power or when the grid electricity is inexpensive. The stored energy can be managed for economic usage in future when the electricity price is high over peak load periods, or when the PV power is unavailable [7]. The grid-connected hybrid system with DSM can help customers to reduce electricity cost, and also can help utility to regulate the grid in terms of security and efficiency issues, such as peak shaving, direct load control (DLC), and capacity market programs [8]. Therefore, at both sides of electricity market hybrid systems may introduce new opportunities to smart grid but also cause many challenges in the following DSM programs.

(1) Peak shaving: it is necessary to decide when and how much to charge the battery from the grid or PV before peak hours, so that power consumed from the grid at peak hours can be reduced to satisfy the requirement of shaving.

(2) Direct load control (in which a utility operator remotely shuts down or cycles a customer's electrical equipment at short notice to address system or local reliability): customers have to control the operation of hybrid system to ensure their demand is satisfied at the shutting time based on the frequency and time of shutting at DLC.

(3) Capacity market programs (in which customers commit to respond pre-specified load reduction when system contingencies arise and are subject 
to penalties if they do not curtail power consumption when directed): such programs involve issues such as how to decide on the amount of power stored in the battery, how to use the instantaneous and stored power cooperatively to complete the pre-specified load reduction, and how to minimize the penalty if the customer demand exceeds the pre-specified demand.

(4) Time-of-use (TOU, where the electricity price is high in the peak load time and low in the off-peak time): scheduling problems arise, such as determining how to optimally operate the hybrid system in peak and off-peak periods for minimizing electricity cost and satisfying the customer demand as well.

It must be noted that the hybrid systems with battery storage may have potential to take part in every DSM program or combined programs, which can help the utility to regulate the grid and help customers to reduce energy cost. For simplicity, this paper will mainly focus on evaluating a gridconnected PV-battery system under the TOU program with contracted selling as an example. It will be answered how customers optimally schedule the hybrid system to earn cost savings with varying prices in the TOU program, and how they manage their consumption to sell surplus power to the grid over peak period.

Although storage systems are not common in large generation farms, for residential and small-scale power producers many storage systems (battery, ultra-capacitor and so on) have been incorporated in energy supply systems. Nair and Garimella [9] argued that battery storage systems will have a significant impact on the small-scale integration of renewable sources into the commercial and residential sectors. For hybrid systems with battery storage, energy management is a vital and difficult issue that has attracted great interest among researchers [7, 10]. Many energy management systems (EMS) have been developed for the utility to regulate microgrids and reduce generation cost. Some rule-based strategies were designed for energy management of hybrid systems $[11,12,13]$, which can obtain promising but not optimal solutions to ensure practical constraints are satisfied. In [14], a deterministic planning method was proposed to perform robustly day-ahead power flow scheduling for conventional and renewable generators. To improve the performance of EMS, optimal control is a useful method to schedule power flows of hybrid systems with minimum cost and maximum benefit $[15,16]$. In [17], an EMS for a virtual power plant was proposed to minimize the electricity generation cost and to utilize renewable energy sufficiently. Authors in [18] presented a dynamic optimal power flow control for power and heat 
generation scheduling while considering PV generations coupled with storage systems. A flexible battery management system was developed to optimize the duration (hours) of charging and discharging battery for optimal power flow control in distribution networks [19].

Beyond existing work, more emphasis should be made on two important issues of renewable hybrid systems. Firstly, most researchers have considered energy management and demand response for large-scale integration of renewable energy at the utility side [20,21]. There is lack of comprehensive work in consideration of optimal planning and DSM for small-scale hybrid systems at the demand side, because many customers install hybrid systems for stand-alone or back-up usage without any participation of DSM program. DSM can be studied more in appliance scheduling of household [22] than in scheduling of small-scale hybrid system. Secondly, uncertainties within forecast errors of renewable energy and demand have been studied for large-scale integration of renewable energy [23], but uncertainties at the demand side are not well evaluated. Most related optimal scheduling methods cannot handle complicated cases when hybrid systems experience external disturbances; only a few closed-loop control methods have been designed [7, 24]. Therefor, it is necessary to model the small-scale hybrid system, to comprehensively study optimal schedule with DSM over different seasons, and to analyze uncertainty and robustness for the closed-loop control. This paper will be organized to respond to the above two issues.

Some remote areas, where customers used to rely on stand-alone hybrid systems for generating power, are being connected to the grid as part of network upgrade. Now a new problem is how to use such installed smallscale system efficiently. Based on our previous work [16], we consider DSM, scheduling and uncertainty handling of the grid-connected hybrid system in this paper. The diesel generator is now excluded, as the power its power generation is less green and more costly than the grid. DSM of the hybrid system is expected to help customers earn some payback and reduce electricity cost. Another by-product advantage of DSM is the reduction of emissions by utilization of clean PV technologies.

The main contributions of this paper are listed below. Firstly, as an example of DSM, the hybrid system under TOU with power selling is modeled to minimize the electricity cost while matching the customer demand and the PV output. Secondly, optimal control is developed as an open loop method to dispatch power flows of the hybrid system stably and economically. A comprehensive study has been conducted to evaluate different situations over 
weekend and weekday of winter and summer. Thirdly, in case of uncertainties in the PV output and the customer demand, model predictive control (MPC) is applied as the closed-loop control to ensure economic, robust and safe operation of the hybrid system. MPC is a feedback control strategy that uses an explicit model of plant to predict the future response of the plant over a finite horizon. Only "the first part" of the sequence is applied to control at the next state $[25,26]$. MPC has been widely used in the closed-loop control for adaptively changing control variables according to external disturbances $[26,27,28]$. MPC is applied in this work because of its capability to explicitly handle constraints and to adjust the power flows when disturbances occur.

In this paper, an optimal power flow management algorithm of a gridconnected PV-battery hybrid system is developed. The objective is to minimize the electricity cost within the DSM framework by optimal power flow control. Literature review is conducted on energy management of stand-alone and grid-connected systems in Section 2. The structure of the grid-connected PV-battery system and its sub-models are described in Section 3. The mathematic DSM model of the hybrid system is given in Section 4. Some results of the optimal control are discussed in Section 5. In Section 6 based on the steady state model an MPC approach is proposed as the closed-loop control, while the last section is the conclusion.

\section{Literature review}

Hybrid renewable energy systems (HRES) have been studied in recent years on both bottom and up levels, such as system design, installation, operation and maintenance. The related studies mainly include issues of modeling, control and optimization at each level. In [29], various sectors in designing and implementation of HRES were comprehensively reviewed, including configurations, criteria selection, sizing methodologies and control \& energy management. For stand-alone and grid-connected applications, many control systems have been designed into three main categories, i.e., centralized [16, 30], distributed [31], and hybrid control paradigms [32]. Due to scope of this paper, energy management methods (planning, scheduling and control) are introduced in this section. Energy management for smart grids have received considerable attention to achieve several targets, such as balancing of generation and load, minimizing the generation cost, minimizing transmission and distribution losses, preventing grid congestion, provision of ancillary services. 
For the stand-alone application, a rule-based power management strategy was designed to manage power flows among different energy sources and storage units [33]. A renewable micro-grid including a wind turbine, a solar panel, a fuel cell and a storage battery was studied on the issue of optimal scheduling [34], in which mixed-integer linear programming is used to solve their proposed minimization model of generation costs subject to all operation technical constraints. A priority local control algorithm was developed to gain optimal energy management of system loads and battery storage, and therefore provided better energy efficiency and guarantee energy supply for critical loads [35]. In [16], daily energy consumption variations between winter and summer was considered into scheduling stand-alone HRES. The authors had evaluated operational efficiency of the hybrid system over a 24hour period and optimal solutions can be found to reduce the corresponding fuel costs. Finally $73 \%$ to $77 \%$ fuel savings in winter and $80.5 \%$ to $82 \%$ fuel savings in summer can be achieved by the optimal control method. In [30], a switched MPC method was designed for energy dispatching of the same HRES.

For the grid-connected application at the utility side, storage management, economic load dispatch and operation optimization of distributed generations was simplified into a single-objective optimization problem to design a smart energy management system of micro-grid [36], which was solved by a matrix real-coded genetic algorithm. A methodology capable of evaluating the impact of wind generation and load uncertainties, as well as unexpected generation outages was developed [23], in which an EMS integration framework was proposed for power system operation, dispatch, and unit commitment. A hybrid power generation system consisting of PV arrays and fuel cells was studied by a model-based optimal approach [24], in which the power generation cost is minimized. A dynamic supervisory control was proposed to regulate a grid-connected hybrid generation system with versatile power transfer for flexible operation and improvement of power quality [37].

For the grid-connected application considering DSM, energy management strategies from both the demand side and utility side were developed to meet the electricity demand while minimizing the overall operating and environmental costs [38]. By integrating DSM and active management schemes, an EMS was developed for optimizing the smart grid's operation to better explore renewable energy sources and reduce the customer's electricity cost [39]. DSM of distributed generation and storage system was studied as a day-ahead optimization problem by a game theory approach in which each 
active user at the demand side selfishly pursues minimal monetary expense for buying/producing electricity [40]. Different demand response programs were studied in DSM of hybrid systems [41, 42]. Optimal power management was studied on grid-connected PV-battery system for joining peak shaving service base on the dynamic programming method [41]. A heuristic-based Evolutionary Algorithm was developed in smart grid for finding a generalized DSM strategy based on load shifting [42]. Many other computational methods were studied in relative applications. A multi-objective method was applied on a hybrid renewable system for maximizing its contribution to the peak load and minimizing its overall intermittence cost, in which large-scale DSM and DR technologies are also considered [20]. Neural networks were applied to schedule and coordinate distributed generations for active DSM [43].

Especially, an interesting application of HRES and DSM is smart building. The impact of DSM strategies in the penetration of HRES is analyzed at some regional buildings [44]. HRES was studied for DSM and an energy production management strategy was designed for building automation [45]. HERS was considered in an optimal residential load management strategy for real time pricing demand response programs [46]. A smart home controller strategy was designed to enable consumer economic saving and automated demand side management in domestic environment [47]. The event driven controller was designed for optimally scheduling household appliances by binary linear programming. An autonomous appliance scheduling strategy was designed for household energy management based on HRES and DSM [22]. An optimization framework was proposed for integrated analysis of demand response programs with high penetration of plug-in hybrid electric vehicles (PHEVs) and PV from residential customer's perspective as well as utility company's perspective [48].

\section{Description of PV-battery system}

The hybrid system evaluated in this paper consists of PV arrays and battery bank that are both connected to the grid. The output power of the PV array feeds customers' demand directly. If the demand is less than the PV's output, the surplus PV power will be charged into the battery bank. If the demand is larger than the PV's output, the deficient power will be covered by the battery or the grid. The grid plays an important role in the hybrid system for charging the battery and directly supplying customers with 
electricity. The battery can be charged by the grid in the off-peak period, and then discharged in the peak period to save electricity cost. The grid provides electricity directly when the customer demand cannot be satisfied by the PV and the battery. The schematic of this hybrid system is shown in Figure 1, in which arrows represent directions of power flows in the system. $P_{1}$ is the solar generation for charging the battery; $P_{2}$ is the discharging power of battery for load demand; $P_{3}$ is the grid power for charging the battery; $P_{4}$ is the grid power for load demand; $P_{5}$ is the solar generation for load demand; $P_{6}$ is the battery discharge for selling power to the grid. In the hybrid system, several converters such as direct current/alternating current (DC/AC) and DC/DC are required for voltage and current matching.

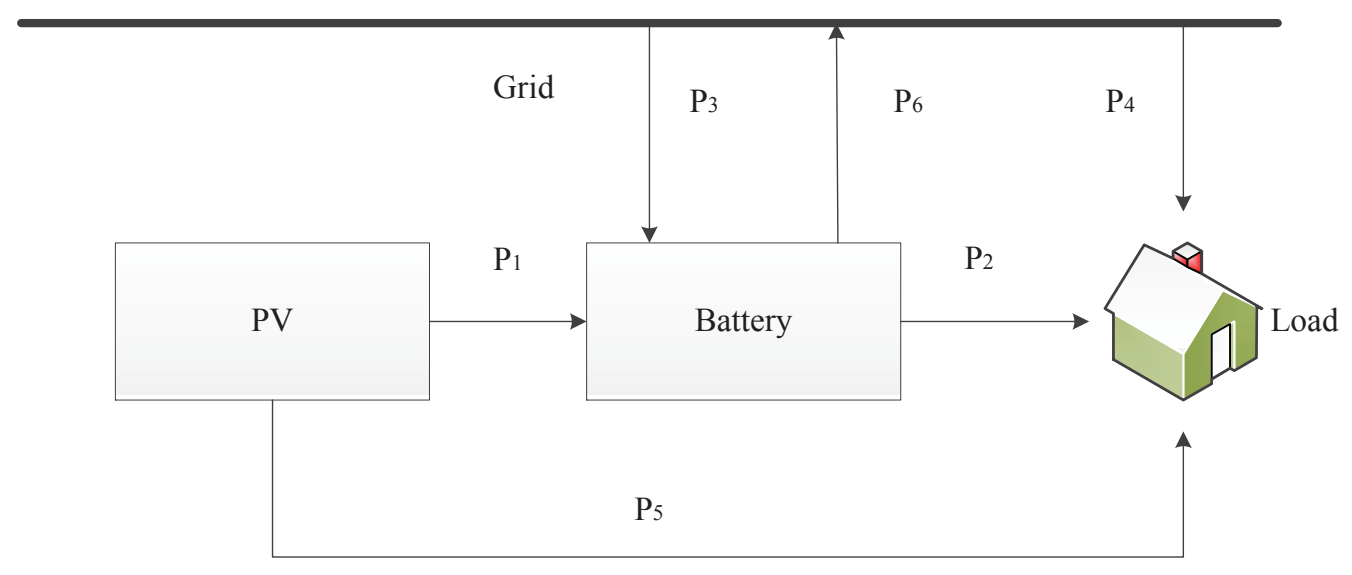

Figure 1: Schematic of the hybrid system

\subsection{PV array}

Each solar array consists of several solar cells to convert sunlight into DC power. The hourly power output of a given area can be simply formulated as:

$$
P_{p v}=\eta_{p v} I_{p v} A_{c}
$$

where $P_{p v}$ is the hourly power output from the PV array; $A_{c}$ is the size of PV array; $\eta_{p v}$ is the efficiency of power generation; $I_{p v}$ is the hourly solar irradiation incident on the PV array $\left(\mathrm{kWh} / \mathrm{m}^{2}\right)$.

The hourly solar irradiation incident on the PV array is closely related to time of a day, season of the year, tilt, location, global irradiation, diffuse 
fraction etc. In this study, the simplified isotropic diffuse formula [16, 49] is used as

$$
I_{p v}=\left(I_{B}+I_{D}\right) R_{B}+I_{D}
$$

where $I_{B}$ is the beam component of the hourly global irradiation and $I_{D}$ is the hourly diffuse irradiation respectively. $R_{B}$ is a geometric ratio of the actual irradiation on the tilted plane to the standard irradiation on the horizontal plane.

The efficiency of power generation can be modeled in a complicated formula, which can be expressed as a function of the hourly irradiation $I_{p v}$ and the ambient temperature $T_{A}$ as

$$
\eta_{p v}=\eta_{R}\left[1-\frac{0.9 \beta I_{p v}\left(T_{C 0}-T_{A 0}\right)}{I_{p v 0}}-\beta\left(T_{A}-T_{R}\right)\right],
$$

where $\eta_{R}$ is the PV generator efficiency that is measured at the referenced cell temperature $T_{R}\left(25^{\circ} \mathrm{C}\right) ; \beta$ is the temperature coefficient for cell efficiency (typically $0.004-0.005 /{ }^{\circ} \mathrm{C}$ ); $T_{C 0}$ (typically $45^{\circ} \mathrm{C}$ ) and $T_{A 0}$ (typically $20^{\circ} \mathrm{C}$ ) are the cell and ambient temperatures at nominal operating cell temperature (NOCT) test conditions; $I_{p v 0}$ is the average solar irradiation on the array at the NOCT conditions.

\subsection{Battery bank}

Being constrained in battery capacity, the state of charge (SOC) changes dynamically owing to possible charge by the PV and grid or possible discharge for customer usage. For a given profile of power generation, customers' demand will mostly affect the SOC of battery. Let $t$ denote time of day (hourly), and $P_{b}(t)$ denote the SOC of battery at the $t^{t h}$ hour. Based on the SOC at the previous hour, the dynamic change of SOC can be formulated as

$$
S(t+1)=S(t)+\eta_{C}\left[P_{1}(t)+P_{3}(t)\right]-\frac{1}{\eta_{D}}\left[P_{2}(t)+P_{6}(t)\right],
$$

where $S(t)$ is the SOC at the $t$ th hour; $S(t+1)$ is the SOC at the next hour. $\eta_{C} \leq 1$ and $\eta_{D} \leq 1$ are the coefficients of charging and discharging efficiency. According to Eq. (4), the current SOC $S(t)$ can be expressed by the initial SOC $S(0)$ of a day as

$$
S(t)=S(0)+\eta_{C} \sum_{\tau=0}^{t-1}\left[P_{1}(\tau)+P_{3}(\tau)\right]-\frac{1}{\eta_{D}} \sum_{\tau=0}^{t-1}\left[P_{2}(\tau)+P_{6}(\tau)\right] .
$$


The SOC of a battery has several constraints, such as the maximal allowable capacity and the depth of discharge (DOD). The lower bound of SOC $S^{m i n}$ can be expressed by the DOD as

$$
S^{\min }=(1-\mathrm{DOD}) S^{\max },
$$

where DOD is the depth of discharge; $S^{\max }$ is the maximum capacity of the battery; $S^{\min }$ is the minimum allowable SOC of the battery. The SOC must be bounded within the scale $\left[S^{\min }, S^{\max }\right]$.

\section{DSM Model of PV-battery system}

Optimal schedule of the evaluated hybrid system aims to minimize electricity cost within the framework of DSM. In this paper, the TOU program is a typical program of DSM for consideration, in which the electricity price changes over different periods according to the electricity supply cost, for example a high price for peak load periods, medium price for standard periods and low price for off-peak periods. In our study, the daily electricity price at the target region can be given as

$$
\rho(t)=\left\{\begin{array}{ll}
\rho_{k}, & t \in T_{k}, T_{k}=[7,10) \bigcup[18,20) \\
\rho_{o}, & t \in T_{o}, T_{o}=[0,6) \bigcup[22,24) \\
\rho_{s}, & t \in T_{s}, T_{s}=[6,7) \bigcup[10,18) \bigcup[20,22)
\end{array},\right.
$$

where $r h o_{k}=0.20538 \$ / \mathrm{kWh}$ is the price for the peak load period; $\rho_{o}=$ $0.03558 \$ / \mathrm{kWh}$ is the price for the off-peak period; $\rho_{s}=0.05948 \$ / \mathrm{kWh}$ is the price for the standard period.

The proposed DSM model includes three parts. The first part is the cost of buying electricity from the grid, which is used to afford the load demand and charge the battery. The second part is the income of selling electricity to the grid. The third part is the wearing cost of hybrid system. The total cost can be formulated as,

$$
J=\sum_{t=0}^{23} \rho(t)\left[P_{3}(t)+P_{4}(t)\right]-\sum_{t \in T_{k}} r_{k} \rho_{k} P_{6}(t)+C_{h},
$$

where $r_{k}=0.65$ is the contracted ratio of the peak price $\rho_{k}$ for selling power

during the peak load period. $C_{h}$ is he wearing cost of system during the 
control period, which is formulated as

$$
C_{h}=\sum_{t=0}^{23} a\left[P_{2}(t)+P_{6}(t)\right]+24 b,
$$

where $a$ is the coefficient of battery wearing cost and $b$ is the hourly wearing cost of other components $(a=0.001, b=0.002$ in this paper). In the objective function, control variables $P_{i}(t)(i=1,2, \ldots, 6,0 \leq t<24)$ have to satisfy several constraints:

(1) PV's output constraint: The PV's power for charging the battery and for customers' instantaneous usage must be less than the PV's output power generated, which is mainly related to irradiation and ambient temperature.

$$
P_{1}(t)+P_{5}(t) \leq P_{p v}(t)
$$

(2) Power balance constraint: The load demand of customers must be exactly satisfied by the total power of PV array, the grid and the battery as

$$
P_{2}(t)+P_{4}(t)+P_{5}(t)=P_{L}(t)
$$

where $P_{L}(t)$ is the load demand over the period $[t, t+1)$.

(3) SOC boundary constraint: The SOC of the battery must be less than the battery's capacity $S^{\max }$ and larger than the minimal allowable value $S^{\text {min }}$ as

$$
S^{\min } \leq S(t) \leq S^{\max }
$$

(4) Power flow constraint: For safety and other physical reasons, power flow from each source must be non-negative and less than the maximum allowable value as

$$
0 \leq P_{i}(t) \leq P_{i}^{\max },(i=1,2, \ldots, 6)
$$

where $P_{i}^{\max }$ is the defined maximum power delivered per hour.

(5) SOC terminate state constraint: For the convenience of dispatching power over continuous days, the battery should not be used till the initial $\mathrm{SOC}$ value is reached. In this model, the termination SOC of the battery must be no less than the initial as SOC

$$
S(0) \leq S(24)
$$


It can be noticed that the installation cost is not considered in the model, as the scope is restricted to a discussion of how to control the installed hybrid system in the operational step. Therefore, some important issues related to installation, such as economic analysis and optimal sizing of the hybrid system $[50,51]$, are neglected in our model. The operational costs of the PV and battery are taken as negligible values for the evaluating period, so they are not incorporated in the model.

\section{Optimal control method}

An open-loop optimal control method is used to dispatch the hourly power $P_{i}(i=1, \ldots, 6)$ over a day to minimize the daily electricity cost, Eq. (8), subject to constraints, Eq. (10-14). Because the objective function and constraints are linear, this power flow control problem can be expressed as a linear programming problem as

$$
\min f(x), \text { s.t. }\left\{\begin{array}{l}
A x \leq b \\
A_{e q} x=b_{e q} \\
l b \leq x \leq u b
\end{array},\right.
$$

where $f(x)$ represents the objective function; $A_{e q}$ and $b_{e} q$ are the coefficients related with equality constraints; $A$ and $b$ are the coefficients related with inequality constraints; $l b$ and $u b$ are the lower and upper bounds of variables. These coefficients can be easily deduced according to the proposed model, so the explicit details are omitted here.

\subsection{Control system settings}

The system evaluated is originally installed as the PV-diesel-battery hybrid system for off-grid consumers [16]. Because of enlarged coverage of the

grid, the target region is now connected to the grid. The diesel generator has been excluded from the current system, because buying electricity from the grid is cheaper and greener than using the diesel generator for power generation. The sizing of PV and battery bank is based on a sizing model in [2]. The parameters of this system are listed in Table 1. The maximum power delivered on each flow is defined as $5 \mathrm{~kW}$.

As customers' daily demand changes between summer and winter as well as between weekdays and weekends, four cases are evaluated, i.e., weekdays of summer and winter and weekends of summer and winter, to find the optimal 
Table 1: Parameters of the PV-battery system

\begin{tabular}{l|c}
\hline \hline Nominal battery capacity & $28.8 \mathrm{kWh}$ \\
\hline Battery charge efficiency & $85 \%$ \\
\hline Battery discharge efficiency & $100 \%$ \\
\hline Battery's depth of discharge & $50 \%$ \\
\hline Initial state of charge & $16 \mathrm{kWh}$ \\
\hline PV array's capacity & $7 \mathrm{~kW}$ \\
\hline
\end{tabular}

dispatch solution for each case. The load profiles of customers in the summer and winter are calculated based on survey data, as given in Table 2 [16].

In this study, we have evaluated meteorological data, global irradiation, diffuse irradiation and ambient temperature over the past few years in the target region. For simplicity, the average output profiles in summer and winter are predicted respectively, as plotted in Figure 2. It can be noticed that power output in summer is larger than in winter over the daytime.

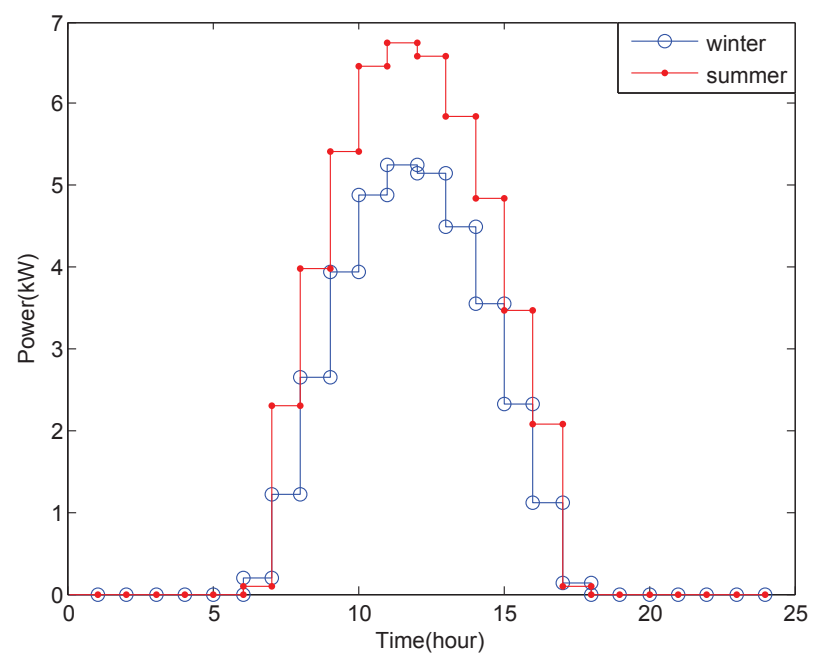

Figure 2: Profiles of hourly power output of the PV array

\subsection{Results of optimal control}

On a winter weekday, without the hybrid system the daily electricity cost would be $\$ 4.27$. When optimally operating the hybrid system the daily electricity cost is reduced to $\$ 1.68$. The income of selling electricity is $\$$ 
Table 2: Demand profiles of four cases

\begin{tabular}{c|l|l|l|l}
\hline \hline \multirow{2}{*}{ Time } & \multicolumn{2}{|c|}{ Winter Load (kW) } & \multicolumn{2}{l}{ Summer Load (kW) } \\
\cline { 2 - 5 } & Weekend & Weekday & Weekend & Weekday \\
\hline $00: 30$ & 1.5 & 1.5 & 1.5 & 1.5 \\
\hline $01: 30$ & 1.5 & 1.5 & 1.5 & 1.5 \\
\hline $02: 30$ & 1.5 & 1.5 & 1.85 & 1.85 \\
\hline $03: 30$ & 1.5 & 1.5 & 1.95 & 1.95 \\
\hline $04: 30$ & 1.5 & 1.5 & 1.85 & 1.85 \\
\hline $05: 30$ & 1.95 & 1.65 & 1.5 & 1.5 \\
\hline $06: 30$ & 1.95 & 1.65 & 1.65 & 1.15 \\
\hline $07: 30$ & 1.65 & 1.35 & 1.65 & 1.25 \\
\hline $08: 30$ & 1.35 & 1.35 & 1.7 & 1.3 \\
\hline $09: 30$ & 3.25 & 3.0 & 1.75 & 1.32 \\
\hline $10: 30$ & 3.25 & 3.0 & 1.75 & 1.35 \\
\hline $11: 30$ & 2.15 & 1.95 & 1.75 & 1.32 \\
\hline $12: 30$ & 2.15 & 1.95 & 1.25 & 1.25 \\
\hline $13: 30$ & 2.15 & 1.95 & 1.32 & 1.32 \\
\hline $14: 30$ & 2.15 & 1.95 & 1.35 & 1.35 \\
\hline $15: 30$ & 2.15 & 1.95 & 1.35 & 1.35 \\
\hline $16: 30$ & 2.15 & 1.65 & 1.45 & 1.45 \\
\hline $17: 30$ & 1.8 & 1.65 & 2.1 & 2.15 \\
\hline $18: 30$ & 2.31 & 3.25 & 2.4 & 2.31 \\
\hline $19: 30$ & 3.81 & 3.25 & 3.8 & 3.25 \\
\hline $20: 30$ & 2.31 & 2.31 & 3.8 & 3.25 \\
\hline $21: 30$ & 2.31 & 2.15 & 2.0 & 2.0 \\
\hline $22: 30$ & 2.31 & 2.15 & 1.95 & 1.95 \\
\hline $23: 30$ & 1.35 & 1.35 & 1.65 & 1.65 \\
\hline & & & \multicolumn{2}{|l}{}
\end{tabular}

3.06. In other words, customers can earn $\$ 1.38$. In Figure 3(a), the optimal power flows at the customer side are plotted for the winter weekday. The customer side power flows include power flows from battery $P_{2}$, grid $P_{4}$ and PV $P_{5}$. During the off-peak period $[0,6)$ and $[22,24)$, only the grid provides power owing to low electricity price. When the PV's output is sufficient, the highest priority is given to the usage of the PV power as shown in the period $[8,16)$. During the peak load period, the power stored in the battery is used to satisfy the load demand. In Figure 3(b), the power flows at the 
battery side are plotted. The battery side power flows include charging flows from PV $P_{1}$ and grid $P_{3}$, and discharging flows to load $P_{2}$ and grid $P_{6}$. The battery is mainly charged from grid during the off-peak periods. When the PV's output is larger than the load demand, the excessive power is stored in the battery. Most battery storage is sold to the grid over the peak load period, and the remaining is used to supply the demand over [18,20). It can be noticed that the SOC increases during the off-peak period and the high irradiation period, and decreases during the peak period. The SOC's boundary constraint and terminate constraint are satisfied.

On a winter weekend, without the hybrid system the daily electricity cost is $\$ 4.47$. When optimally operating the hybrid system, the daily cost of electricity is reduced to $\$ 1.88$, and the income of selling electricity is $\$ 3.11$. The net value earned is $\$ 1.23$. For the winter weekend, the power flows at the customer side are shown in Figure 3(c). It can be noticed that the result is similar to that for the winter weekday. During the off-peak period, customers use the grid power owing to the low electricity price. During certain standard period, although the PV generation can fully satisfy the load demand, the grid power has been used. To store enough power for sale, the battery is not discharged during the standard and off-peak periods. In Figure 3(d), the power flows at the battery side are plotted. Similarly, the battery is charged sufficiently during the off-peak period and this stored power is used during the peak period. During the sufficient irradiation period, the battery is mainly charged by the PV. In this case, constraints related to the SOC are satisfied during the charging and discharging processes.

On a summer weekday, without the hybrid system the daily electricity cost is $\$ 3.49$. When optimally operating the hybrid system, the electricity cost is reduced to $\$ 1.32$, but the income of selling electricity is $\$ 3.18$. This means customers can eventually earn $\$ 1.83$. For the summer weekday, the profiles of power flow at the demand side and the battery side have been given in Figure 4(a) and (b). Because in this case the total demand is the lowest among the evaluated four cases, during the standard period more PV power can be stored in the battery and no grid power is required for charging the battery. Most grid power is used during the off-peak period for satisfying the load demand and charging the battery.

On a summer weekend, without the hybrid system the daily electricity cost is $\$ 3.99$. When optimally operating the hybrid system, the electricity cost is reduced to $\$ 1.41$. The income of selling electricity is $\$ 3.10$, which means customers can earn $\$ 1.69$. As shown in Figure $4(\mathrm{c})$, the grid power 

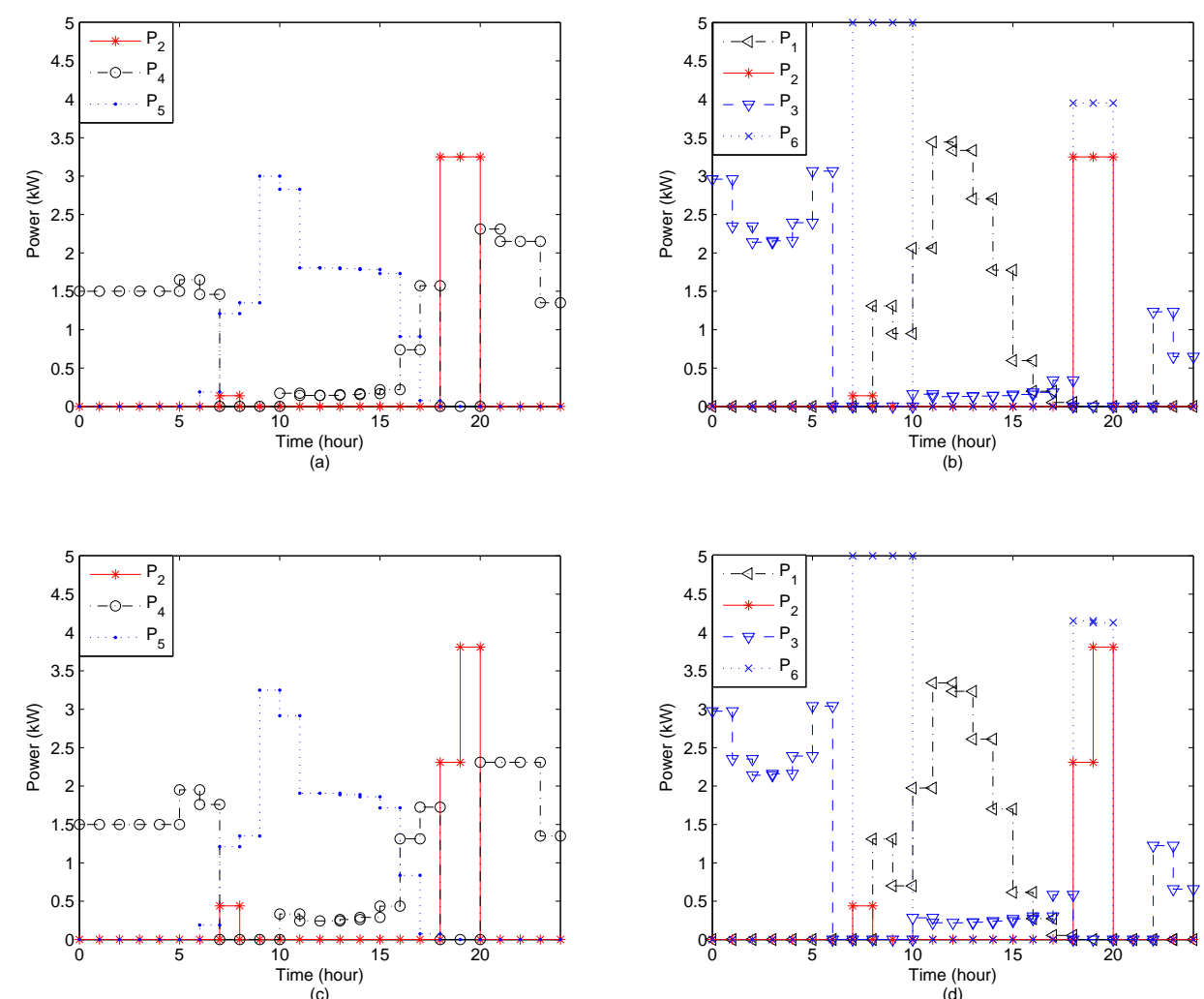

Figure 3: Power flows during winter weekend and weekday: (a) customer side power flows, i.e., from battery, grid and PV to load, for a winter weekday; (b) battery side power flows, i.e., charging flows from grid and PV and discharging flows to load and grid, for a winter weekday; (c) customer side power flows for a winter weekend; (d) battery side power flows for a winter weekday.

is consumed during the off-peak period on the summer weekend. The power flows at the battery side are also shown in Figure 4(d), in which the battery is discharged in the off-peak and standard periods. The results for the summer weekend are almost the same as those for a summer weekday, and their demand profiles are close to each other.

In sum, it is observed that by optimal control the hybrid system the monthly income in winter is $\$ 40.20$, which saves $\$ 170.60$ for customers. The monthly income in summer is $\$ 51.82$, which saves $\$ 167.52$ for the customers. 

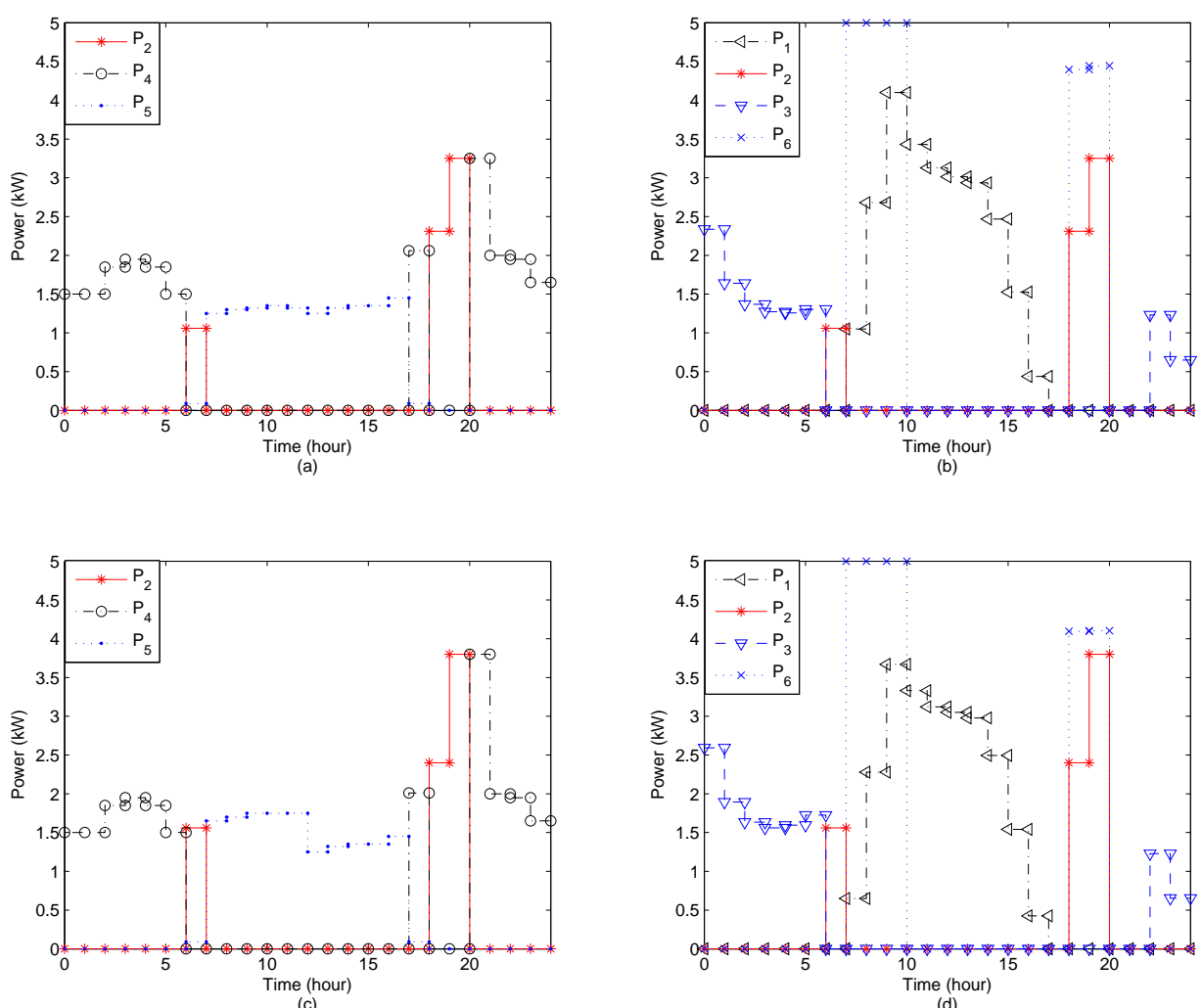

Figure 4: Power flows during summer weekend and weekday: (a) customer side power flows, i.e., from battery, grid and PV to load, for a summer weekday; (b) battery side power flows, i.e., charging flows from grid and PV and discharging flows to load and grid, for a summer weekday; (c) customer side power flows for a summer weekend; (d) battery side power flows for a summer weekday.

Because load demand in winter is larger than that in summer, the monthly earn in winter is less than that in summer, but the total cost savings are almost the same after optimally scheduling.

Over a year period study, load demand and PV output can be forecast requently on daily or weekly basis, as during a year they are varying largely due to weather and human factors. Different load demand and PV output have significant effects on operation hours and cost saving. Although we have not given actual statistics for a whole year, based on the summer and 
winter results the total income of selling electricity is approximately $\$ 552.12$ and the total cost saving is approximately $\$ 2028.72$. For a newly installed PV-battery hybrid system, the capital cost of installation is $\$ 12500$, and its yearly operation and maintenance cost is $\$ 135$. The payback period is about 6.6 years. For an existing PV-battery hybrid system, the capital cost of installation is omitted and the payback period of modification must be less than 1 year.

\section{Model predictive control method}

In the optimal control, customer demand loads during weekdays and weekends are forecast as the average values for winter or summer; the profile of PV output is also forecast as the average values for winter and summer. In fact, the customer demand and the PV output may be disturbed from the forecast values. In this section, such divergence is evaluated as system disturbances on the demand and the PV output. The linear state-space model will be deduced from the hybrid system model. From Eq. (11), the following equation can be obtained

$$
P_{4}(t)=P_{L}(t)-P_{2}(t)-P_{5}(t)
$$

Then denote the control input as $u(t) \triangleq\left[P_{1}(t), P_{2}(t), P_{3}(t), P_{5}(t), P_{6}(t)\right]^{T}$, the system state as $x(t) \triangleq S(t)$ and the output as $y(t) \triangleq P_{4}(t)$. From Eq. $(5)$, the linear state-space model with disturbances can be expressed as

$$
\begin{cases}x(t+1) & =A x(t)+B u(t)+B w(t) \\ y(t) & =C x(t)+D u(t)+D w(t)+P_{L}(t)+w_{L}(t)\end{cases}
$$

where $A=I, C=0, B=\left[\eta_{C},-\eta_{D}, \eta_{C}, 0,-\eta_{D}\right], D=[0,-1,0,0,-1,0] ; w(t)$ and $w_{L}(t)$ are disturbances of input and output respectively. Although $w(t)$ is caused by differences of predicted and actual values in terms of the customer load and the PV output, it is uneasy to determine $w(t)$ according to these differences. If the customer demand experiences disturbance $w_{L}(t)$ and the PV output experiences disturbance $w_{p v}(t)$ respectively, the adjusted disturbance $w(t)$ is determined using the following proposed rules, in which $P_{i}(t), i=1,2, \ldots, 6$ are pre-scheduled power flows without consideration of disturbance.

(a) If $w_{p v}(t)$ is negative, reduce $P_{1}(t)$ firstly to satisfy the PV's output constraint. If $P_{1}(t)=0$, then reduce $P_{5}(t)$ till the constraint is satisfied. 
(b) For a positive $w_{p v}(t)$, increase $P_{1}(t)$ as $P_{1}(t)=P_{1}(t)+w_{p v}(t)$ if $w_{L}(t) \leq$ 0 ; else increase $P_{5}(t)$ as $P_{5}(t)=P_{5}(t)+w_{p v}(t)$.

(c) If $P_{2}(t)+P_{5}(t)>P_{L}(t)+w_{L}(t)$, reduce $P_{2}(t)$ firstly to satisfy the power balance constraint. If $P_{2}(t)=0$, then reduce $P_{5}(t)$ till the power balance constraint is satisfied.

(d) If $P_{2}(t)+P_{5}(t)<P_{L}(t)+w_{L}(t)$, increase $P_{2}(t)$ by $\alpha\left[w_{L}(t)-\Delta P_{2}(t)-\Delta P_{5}(t)\right]$, where $\Delta P_{2}(t)$ and $\Delta P_{5}(t)$ denote increments of $P_{2}(t)$ and $P_{5}(t)$ during steps (a-c).

(e) Bound $P_{i}(t)(i=1,2,5)$ within $\left[0, P_{i}^{\max }\right]$. If $S(t-1)+\eta_{C} P_{1}(t)-$ $P_{2}(t) / \eta_{D}-P_{6}(t) / \eta_{D} \leq S^{\text {max }}$ is not satisfied, reduce $P_{1}(t)$ firstly to satisfy this condition, then if $P_{1}(t)=0$ continue to increase $P_{2}(t)$ till the condition is satisfied. If $S(t-1)+\eta_{C} P_{1}(t)+\eta_{C} P_{3}^{\max }-P_{2}(t) / \eta_{D}-P_{6}(t) / \eta_{D} \geq S^{\min }$ is not satisfied, reduce $P_{2}(t)$ to satisfy this condition. If the $S O C$ cannot satisfy the boundary constraint, reduce $P_{3}(t)$ if $S(t)>S^{\max }(t)$; increase $P_{3}(t)$ if $S(t)<S^{\min }(t)$.

(f) The actual output is calculated according to Eq. (16).

Note that in (a) and (b), $P_{5}(t)$ has higher priority of usage than $P_{1}(t)$ because the charging or discharging processes cause loss of energy. In (c), the battery is seldom employed if $P_{5}(t)$ is sufficient to provide power. In (d), when the power balance at the customer side is broken, the battery takes some responsibility for covering the disturbed load demand. The responsibility rate $\alpha$ is an ad-hoc parameter. In this paper, $\alpha=35 \%$ is used during the standard period, $\alpha=50 \%$ is used during the peak period, $\alpha=20 \%$ is used during the off-peak period. In (e), $P_{1}(t), P_{2}(t), P_{3}(t)$ are re-adjusted when the SOC boundary constraint is violated. At the last step, the output is determined by the actual load demand and the system input. The rational behind the rule is that PV power for customer usage has higher efficiency that PV power for storage, and that load demand balance is mainly satisfied by the grid power supply and then by the battery discharge. When the demand increases, the battery will discharge more over the off-peak period than the peak period for the price concern

MPC is developed for the closed-loop control, in which the objective function of DSM model is optimized over the receded prediction horizon. Combining Eq. (8) and (16), the objective function over the prediction horizon 
$T_{N}=\left[k, k+N_{p}\right)(k \geq 0$ is an integer $)$ is obtained as follows:

$$
\begin{aligned}
J_{c}=\sum_{t=k}^{k+N_{p}-1} \rho(t)\left[P_{L}(t)\right. & \left.+P_{3}(t)-P_{2}(t)-P_{5}(t)\right] \\
& -\sum_{t \in T_{k} \cap T_{N}} r_{k} \rho_{k} P_{6}(t)+C_{h} .
\end{aligned}
$$

where $N_{p}$ is hours over the prediction horizon, and the wearing cost of battery over the prediction horizon is $C_{B}=\sum_{t=k}^{k+N_{p}-1} a\left[P_{2}(t)+P_{6}(t)\right]+N_{p} b$. As in Eq. (10-15), the constraints of MPC can be expressed as

$$
\left\{\begin{array}{l}
P_{1}(t)+P_{5}(t) \leq P_{p v}(t) \\
S^{\min } \leq S(t) \leq S^{\max } \\
0 \leq P_{i}(t) \leq P_{i}^{\max }, i=1,2,3,5,6 \\
0 \leq P_{L}(t)-P_{2}(t)-P_{5}(t) \leq P_{4}^{\max } \\
S(0) \leq S(N)
\end{array}\right.
$$

where $N$ is hours over the overall scheduling period. Note that $S(0) \leq S(N)$ is only valid in the MPC approach when $k \geq N-N_{p}+1$.

MPC is employed to solve this optimal control problem at each sampling period. In the proposed MPC approach, an optimal control problem over the prediction horizon is repeatedly solved $\left(k=0, \ldots, N-N_{p}\right)$. The optimal control problem, including the objective function and the set of constraints, has been defined in Eq. (18) and (19). The optimization variable is the power flow sequence at each sampling period. At the $k$ th sample, an optimal solution $\left[\bar{u}(k), \bar{u}(k+1), \ldots, \bar{u}\left(k+N_{p}-1\right)\right]^{T}$ can be obtained after solving the optimal problem. Only the first part of solution, i.e., $\bar{u}(k)$, is used in the current period. According to our proposed rules, the disturbance of the input $w(k)$ applied to the system in the period $[k, k+1)$ can be determined. When the planning horizon gets shorter than the prediction horizon $N_{p}$, i.e. $k>N-N_{p}+1$, the prediction period will be decreased by 1 after each sample. The procedure of the MPC approach can be illustrated as in Algorithm 1. The schematic of MPC is illustrated in Figure 5. At the $k$ th sampling instant, the feedback state is firstly measured, and the control input $u(k)$ is computed as Algorithm 1. Then control input is implemented on the open loop plant for system operation. Note that the main difference between the open loop control and MPC is that the open loop control does not have the feedback and the control input is pre-designed off-line, but MPC has the feedback and real time control mechanisms. 


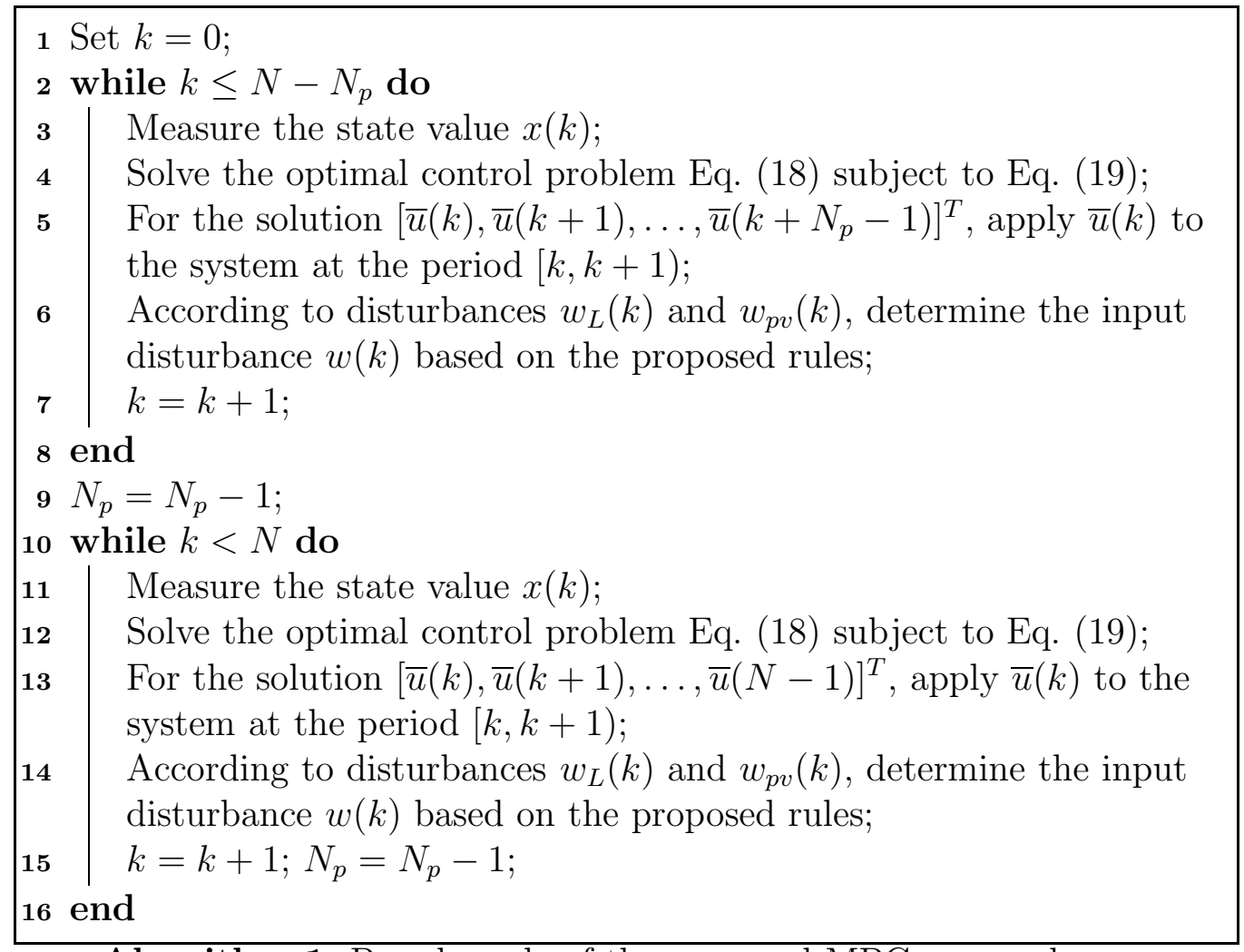

Algorithm 1: Pseudo-code of the proposed MPC approach 


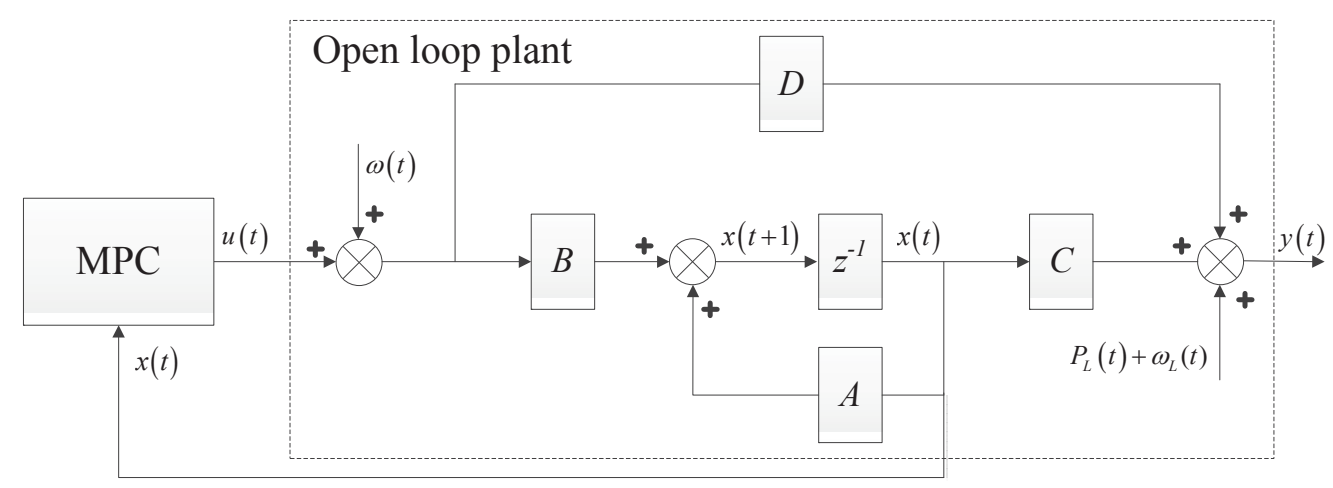

Figure 5: Schematic of the MPC approach

To verify the performance of MPC, three experiments with various disturbances are evaluated. The sampling period is one hour. The scheduling period is five workdays in winter, and the prediction period is 24 hours. This closed-loop control is compared with the open loop control (as illustrated in the previous section) regarding cost saving. For simplification, a distribution fitting method [23] is used as our uncertainty model of disturbance in this paper. The distribution fitting method includes a hypothesis regarding a standard probability distribution of the forecast error (solar generation or load) and a fitting procedure used to find its parameters. Load and solar forecast errors are assumed to follow truncated normal distribution (TND). Probability density function (PDF) of TND is

$$
P D F_{T N D}(x)=\frac{\frac{1}{\sigma} P D F_{N}\left(\frac{x-\mu}{\sigma}\right)}{C D F_{N}\left(\frac{b-\mu}{\sigma}\right)-C D F_{N}\left(\frac{a-\mu}{\sigma}\right)},
$$

where $\mu$ is the mean value of non-truncated normal distribution; $\sigma$ is standard deviation of non-truncated normal distribution; $a$ and $b$ are upper and lower limits of TND, i.e., $x \in(a, b), a<b ; P D F_{N}$ is the PDF of standard normal distribution; and $C D F_{N}$ is cumulative distribution function of standard normal distribution.

(1) Positive disturbance on PV output: In this experiment, the solar irradiation is assumed to be larger than the predicted irradiation. Then the PV output experiences positive disturbance. For an hour, the positive disturbance is assumed to follow TND with parameters $\mu=0, \sigma=0.4, a=$ $0, b=1.5$, which are obtained by analyzing historical data. 
In the MPC approach, it is desired that the additional PV output disturbance can be used to charge the battery, so that the customer will use less power from the grid. The resulted profiles of $P_{2}$ and $P_{4}$ are plotted in Figure $6(\mathrm{a})$ and (b). It can be noticed that more solar power is used to charge the battery in the closed-loop than in the open loop control. It is also observed that the customer needs less power from the grid in the closed-loop. The net ncome for five days is $\$ 1.02$ for open-loop control and $\$ 1.14$ for MPC. The earning has increased about $27 \%$ after using MPC.
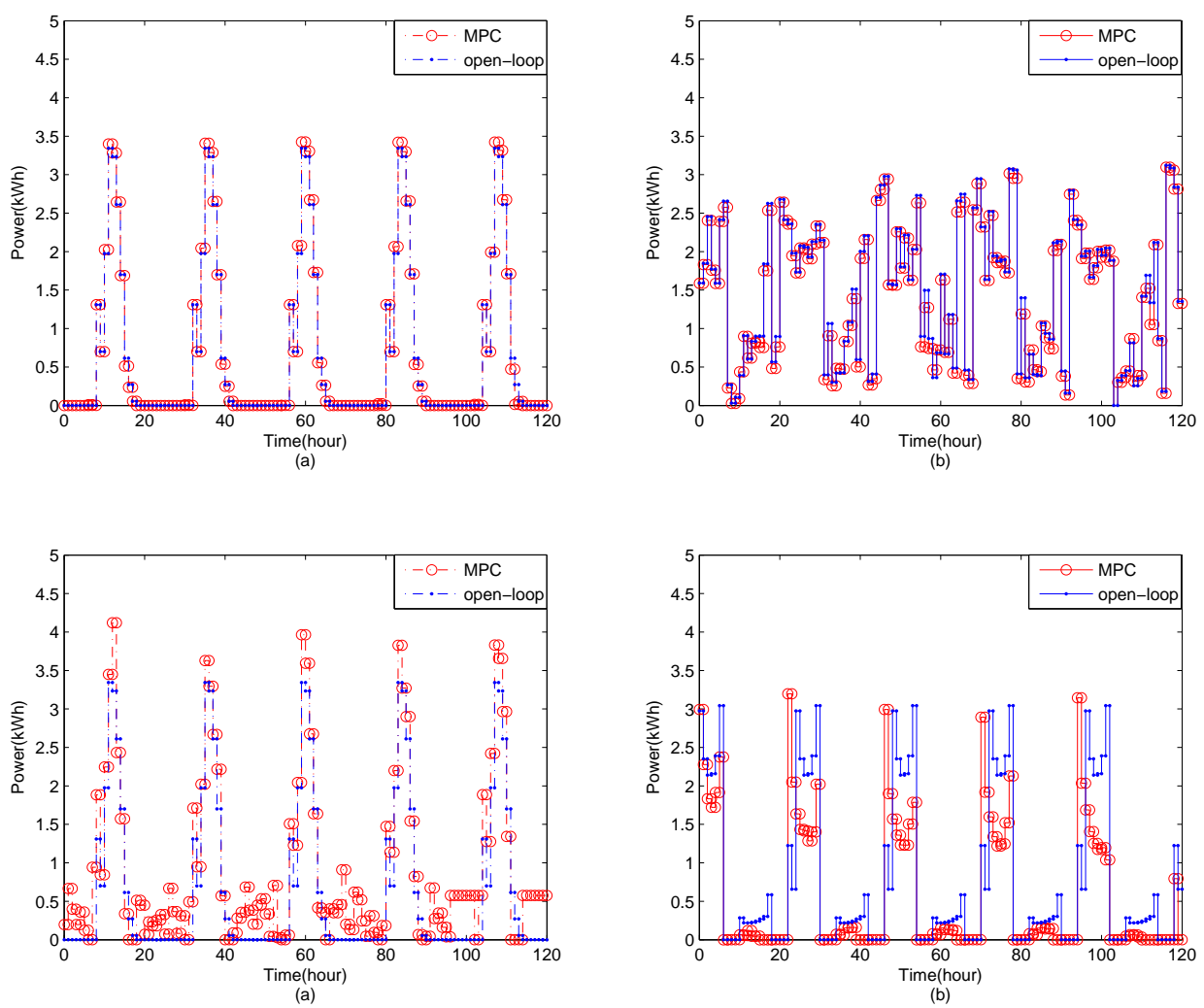

Figure 6: Power flows in Experiment 1 and 2: (a) $P_{1}$ of Experiment 1; (b) $P_{4}$ of Experiment $1 ;$ (c) $P_{2}$ of Experiment 2; (d) $P_{3}$ of Experiment 2

(2) Positive disturbance on customer demand:

In the second experiment, customers are assumed to demand more power 
than the predicted value. Then the customer demand experiences positive disturbance. For an hour, the positive disturbance is assumed follow TND with parameters $\mu=0, \sigma=0.6, a=0, b=1.5$, which are obtained by analyzing historical data. For the open-loop control, the amount of additional demand asks for the same amount of additional power from the grid, i.e., increasing $P_{4}$.

In the closed-loop control, the battery takes predefined responsibility to provide power to satisfy the additional disturbance on demand. To achieve this task, the battery must be charged sufficiently by the grid power over the off-peak period to reduce the electricity cost. The power loss during the charging process must be considered in the cost at the same time. Figure 6(c) and (d) show the profiles of $P_{2}$ and $P_{3}$ for both MPC and open-loop control. An interesting observation is that the battery discharges more in the peak and standard periods (as shown in (c)) and more grid power is used to charge the battery over the off-peak time $(22,24$ ] (as shown in (d)). As a result, the net income for five days is $\$ 7.89$ for MPC compared with $\$ 6.01$ for open-loop control. It can be noticed that the earning increases around $31 \%$ after using MPC.

(3) Random disturbances on PV output and demand: In the third experiment, the disturbances are random numbers, which can be positive or negative. The random disturbances follow TND with parameters $\mu_{1}=0, \sigma_{1}=$ $0.4, a_{1}=-1.5, b_{1}=1.5$ for solar forecast error and $\mu_{2}=0, \sigma_{2}=0.6, a_{2}=$ $-1.5, b_{2}=1.5$ for load forecast error.

It is obvious that open-loop control cannot handle such a complicated case because the PV output and the load balance constraints are not satisfied. However, by using the MPC approach, all constraints can be satisfied and the electricity cost is also optimized. To compare effects of disturbances, the MPC approach is also implemented on the hybrid system without consideration of disturbance, in which the obtained results are called nominal values. The profiles of SOC obtained by MPC during five days are given in Figure 7, in which the profile under disturbances is compared with the nominal profile under no disturbance. By using the MPC approach, the battery SOC is maintained within a safe range, and the SOC under random disturbances converges to the nominal value. The robustness of MPC is achieved owing to its closed-loop mechanism. The net income in this case is still promising as $\$ 4.83$. 


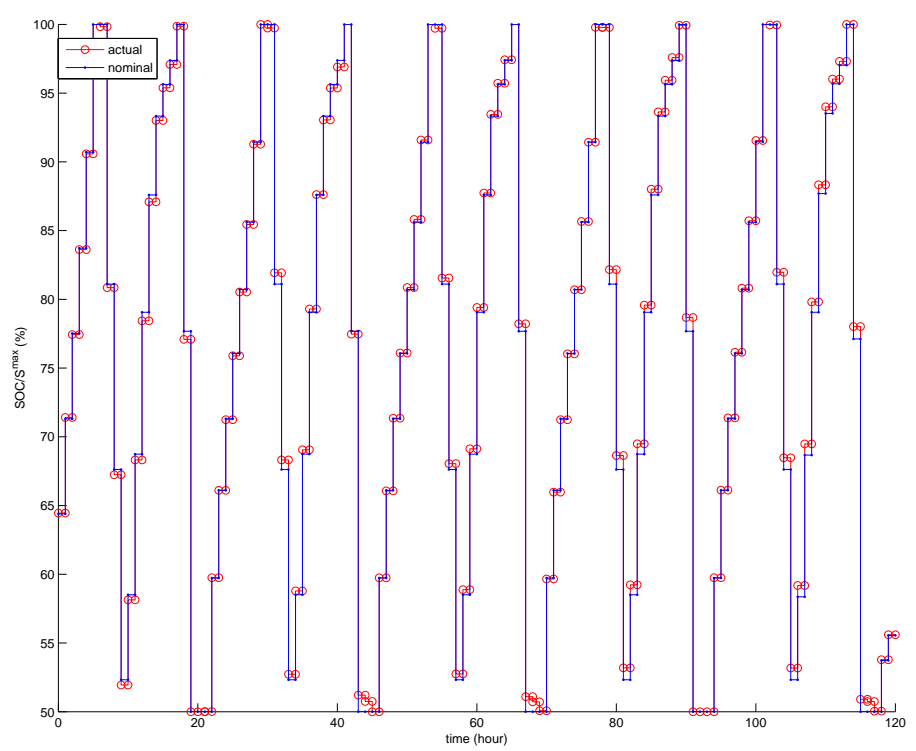

Figure 7: Profile of SOC for Experiment 3

\section{Conclusion}

Demand side management has been considered in the optimal scheduling of small-scale PV-battery hybrid system on the behalf of customers. An example of DR program, i.e., TOU with power selling over peak period, has been studied for energy management in this paper. A model for reducing electricity cost has been developed, which is much practical at the customer side. The results have shown that the optimal solution to the operation of hybrid system achieves the maximal use of solar energy and battery storage. It can be observed that the battery plays a significant role in storing grid power during off-peak periods and supplying power to customers during peak periods. As a result, by scheduling the hybrid system, customers consume minimal amount of power from the grid and reduce their monthly cost. It has been shown that optimal control is a useful open-loop control method for power flow control in DSM.

As that open loop control cannot handle the control task when the hybrid system experiences disturbances in PV output and load demand, MPC has been developed for controlling such a hybrid system when disturbances occur. 
For this closed-loop control method, the linear state-space model has been formulated. The proposed MPC approach has been employed to schedule the disturbed hybrid system. Closed-loop control has been compared with open loop control in the presented simulation study. Any disturbance experienced in the system can be detected before the next control period, and the control variable can be corrected accordingly for the next period by employing the MPC approach. For a highly disturbed system that cannot be handled by open loop control, MPC can achieve great control performance in terms of accuracy and robustness. Furthermore, more cost savings can be obtained by using the closed-loop control.

In this work, only TOU is evaluated in the small-scale hybrid system as an example of DSM. Future work include considering other DSM programs and extending the model to incorporate more renewable energy sources such as wind power, biomass power, hydro power and so on.

\section{References}

[1] Shaahid S, El-Amin I. Techno-economic evaluation of off-grid hybrid photovoltaic-diesel-battery power systems for rural electrification in saudi arabia - a way forward for sustainable development. Renewable and Sustainable Energy Reviews 2009;13(3):625 -33.

[2] Hove T, Tazvinga H. A techno-economic model for optimising component sizing and energy dispatch strategy for PV-diesel-battery hybrid power systems. Journal of Energy in Southern Africa 2012;23(4):18 28.

[3] Arun P, Banerjee R, Bandyopadhyay S. Optimum sizing of photovoltaic battery systems incorporating uncertainty through design space approach. Solar Energy 2009;83(7):1013 -25.

[4] Giraud F, Salameh Z. Steady-state performance of a grid-connected rooftop hybrid wind-photovoltaic power system with battery storage. IEEE Trans on Energy Conversion 2001;16(1):1-7.

[5] Gabash A, Li P. Flexible optimal operation of battery storage systems for energy supply networks. IEEE Trans on Power Systems $2013 ; 28(3): 2788-97$. 
[6] Kanchev H, Lu D, Colas F, Lazarov V, Francois B. Energy management and operational planning of a microgrid with a PV-based active generator for smart grid applications. IEEE Trans on Industrial Electronics 2011;58(10):4583-92.

[7] Palma-Behnke R, Benavides C, Lana F, Severino B, Reyes L, Llanos $\mathrm{J}$, et al. A microgrid energy management system based on the rolling horizon strategy. IEEE Trans on Smart Grid 2013;4(2):996 - 1006.

[8] Aalami H, Moghaddam MP, Yousefi G. Demand response modeling considering interruptible/curtailable loads and capacity market programs. Applied Energy 2010;87(1):243 -50.

[9] Nair NKC, Garimella N. Battery energy storage systems: Assessment for small-scale renewable energy integration. Energy and Buildings 2010;42(11):2124-30.

[10] Levron Y, Guerrero J, Beck Y. Optimal power flow in microgrids with energy storage. IEEE Trans on Power Systems 2013;28(3):3226-34.

[11] Wang C, Nehrir M. Power management of a stand-alone wind/photovoltaic/fuel cell energy system. IEEE Trans on Energy Conversion 2008;23(3):957-67.

[12] Jain S, Agarwal V. An integrated hybrid power supply for distributed generation applications fed by nonconventional energy sources. IEEE Trans on Energy Conversion 2008;23(2):622-31.

[13] Teleke S, Baran M, Bhattacharya S, Huang A. Rule-based control of battery energy storage for dispatching intermittent renewable sources. IEEE Trans on Sustainable Energy 2010;1(3):117-24.

[14] Kanchev H, Lu D, Colas F, Lazarov V, Francois B. Energy management and operational planning of a microgrid with a PV-based active generator for smart grid applications. IEEE Trans on Industrial Electronics 2011;58(10):4583-92.

[15] Riffonneau Y, Bacha S, Barruel F, Ploix S. Optimal power flow management for grid connected PV systems with batteries. IEEE Trans on Sustainable Energy 2011;2(3):309-20. 
[16] Tazvinga H, Xia X, Zhang J. Minimum cost solution to photovoltaicdiesel-battery hybrid power systems for remote consumers. Solar Energy 2013;96:292 -9.

[17] Lombardi P, Powalko M, Rudion K. Optimal operation of a virtual power plant. In: Power Energy Society General Meeting, 2009. PES '09. IEEE. 2009, p. 1-6.

[18] Derakhshandeh S, Masoum A, Deilami S, Masoum M, Hamedani Golshan M. Coordination of generation scheduling with PEVs charging in industrial microgrids. IEEE Trans on Power Systems 2013;28(3):345161.

[19] Gabash A, Li P. Flexible optimal operation of battery storage systems for energy supply networks. IEEE Trans on Power Systems 2013;28(3):2788-97.

[20] Moura PS, de Almeida AT. Multi-objective optimization of a mixed renewable system with demand-side management. Renewable and Sustainable Energy Reviews 2010;14(5):1461 -8.

[21] Huang L, Walrand J, Ramchandran K. Optimal demand response with energy storage management. In: 2012 IEEE Third International Conference on Smart Grid Communications (SmartGridComm). 2012, p. $61-6$.

[22] Adika CO, Wang L. Autonomous appliance scheduling for household energy management. IEEE Trans on Smart Grid 2014;5(2):673 -82.

[23] Makarov Y, Etingov P, Ma J, Huang Z, Subbarao K. Incorporating uncertainty of wind power generation forecast into power system operation, dispatch, and unit commitment procedures. IEEE Transactions on Sustainable Energy 2011;2(4):433-42.

[24] Zervas P, Sarimveis H, Palyvos J, Markatos N. Model-based optimal control of a hybrid power generation system consisting of photovoltaic arrays and fuel cells. Journal of Power Sources 2008;181(2):327 -38.

[25] García CE, Prett DM, Morari M. Model predictive control: Theory and practicea survey. Automatica 1989;25(3):335 -48. 
[26] Xia X, Zhang J, Elaiw A. An application of model predictive control to the dynamic economic dispatch of power generation. Control Engineering Practice 2011;19(6):638 -48.

[27] Zhang J, Xia X. A model predictive control approach to the periodic implementation of the solutions of the optimal dynamic resource allocation problem. Automatica 2011;47(2):358 -62.

[28] Wu Z, Xia X. Model predictive control for improving operational efficiency of overhead cranes. Nonlinear Dynamics 2014;:1 - 19.

[29] Upadhyay S, Sharma M. A review on configurations, control and sizing methodologies of hybrid energy systems. Renewable and Sustainable Energy Reviews 2014;38:47 - 63.

[30] Zhu B, Tazvinga H, Xia X. Switched model predictive control for energy dispatching of a photovoltaic-diesel-battery hybrid power system. IEEE Trans on Control Systems Technology 2014;doi:10.1109/TCST.2014.2361800.

[31] Lagorse J, Simoes M, Miraoui A. A multiagent fuzzy-logic-based energy management of hybrid systems. IEEE Transactions on Industry Applications 2009;45(6):2123-9.

[32] Torreglosa J, Garca P, Fernndez L, Jurado F. Hierarchical energy management system for stand-alone hybrid system based on generation costs and cascade control. Energy Conversion and Management 2014;77:514 -26 .

[33] Wang C, Nehrir M. Power management of a stand-alone wind/photovoltaic/fuel cell energy system. IEEE Transactions on Energy Conversion 2008;23(3):957-67.

[34] Morais H, Kádár P, Faria P, Vale ZA, Khodr H. Optimal scheduling of a renewable micro-grid in an isolated load area using mixed-integer linear programming. Renewable Energy 2010;35(1):151 -6.

[35] Faxas-Guzmán J, García-Valverde R, Serrano-Luján L, Urbina A. Priority load control algorithm for optimal energy management in stand-alone photovoltaic systems. Renewable Energy 2014;68:156 -62. 
[36] Chen C, Duan S, Cai T, Liu B, Hu G. Smart energy management system for optimal microgrid economic operation. IET Renewable Power Generation 2011;5(3):258-67.

[37] Kim SK, Jeon JH, Cho CH, Ahn JB, Kwon SH. Dynamic modeling and control of a grid-connected hybrid generation system with versatile power transfer. IEEE Transactions on Industrial Electronics 2008;55(4):1677-88.

[38] Wang X, Palazoglu A, El-Farra NH. Operational optimization and demand response of hybrid renewable energy systems. Applied Energy $2015 ; 143: 324-35$.

[39] Cecati C, Citro C, Siano P. Combined operations of renewable energy systems and responsive demand in a smart grid. IEEE Transactions on Sustainable Energy 2011;2(4):468-76.

[40] Atzeni I, Ordonez L, Scutari G, Palomar D, Fonollosa J. Demand-side management via distributed energy generation and storage optimization. IEEE Transactions on Smart Grid 2013;4(2):866-76.

[41] Riffonneau Y, Bacha S, Barruel F, Ploix S. Optimal power flow management for grid connected PV systems with batteries. IEEE Transactions on Sustainable Energy 2011;2(3):309-20.

[42] Logenthiran T, Srinivasan D, Shun TZ. Demand side management in smart grid using heuristic optimization. IEEE Transactions on Smart Grid 2012;3(3):1244-52.

[43] Matallanas E, Castillo-Cagigal M, Gutiérrez A, Monasterio-Huelin F, no Martín EC, Masa D, et al. Neural network controller for active demandside management with $\{\mathrm{PV}\}$ energy in the residential sector. Applied Energy 2012;91(1):90 -7.

[44] Pina A, Silva C, ao PF. The impact of demand side management strategies in the penetration of renewable electricity. Energy 2012;41(1):128 -37 .

[45] Figueiredo J, Martins J. Energy production system management - renewable energy power supply integration with building automation system. Energy Conversion and Management 2010;51(6):1120 -6. 
[46] Lujano-Rojas JM, Monteiro C, Dufo-López R, Bernal-Agustín JL. Optimum residential load management strategy for real time pricing (RTP) demand response programs. Energy Policy 2012;45(0):671 -9.

[47] Giorgio AD, Pimpinella L. An event driven smart home controller enabling consumer economic saving and automated demand side management. Applied Energy 2012;96:92 - 103.

[48] Zhao J, Kucuksari S, Mazhari E, Son YJ. Integrated analysis of highpenetration PV and PHEV with energy storage and demand response. Applied Energy 2013;112:35 - 51.

[49] Collares-Pereira M, Rabl A. The average distribution of solar radiation correlation between diffuse and hemispherical and between daily and hourly insolation values. Solar Energy 1979;22:155 -64.

[50] Yang H, Wei Z, Chengzhi L. Optimal design and techno-economic analysis of a hybrid solar-wind power generation system. Applied Energy 2009;86(2):163-9.

[51] Roy A, Kedare SB, Bandyopadhyay S. Optimum sizing of windbattery systems incorporating resource uncertainty. Applied Energy 2010;87(8):2712-27. 